\title{
EFECTO DEL MOMENTO FLEXIONANTE EN LA FUERZA CORTANTE QUE PRODUCE EL AGRIETAMIENTO POR TENSIÓN DIAGONAL EN MUROS DE MAMPOSTERÍA CONFINADA
}

\author{
Juan José Pérez Gavilán. ${ }^{1}$ y Antonio Manzano².
}

\begin{abstract}
RESUMEN
En este estudio se establece que la presencia de momento flexionante en muros de mampostería confinada tiene un efecto en la fuerza cortante que produce el primer agrietamiento por tensión diagonal. Se propone a priori una hipótesis que relaciona la distorsión lateral con la aparición del agrietamiento y con base en ella se desarrolla una expresión matemática que predice el cortante que produce el agrietamiento por tensión diagonal cuando se aplica un momento flexionante en el extremo superior del muro. Posteriormente se presentan los resultados de un estudio experimental piloto consistente en dos muros de mampostería confinada a escala natural: uno probado con fuerza cortante y carga axial, que sirvió de referencia y otro probado con momento, carga axial y fuerza lateral creciente. Se encontró, en el segundo caso, una reducción en la fuerza cortante que produjo el agrietamiento por tensión diagonal, en buen acuerdo con lo predicho por el modelo matemático propuesto. Se hacen algunas observaciones y se dan recomendaciones para la realización de un estudio más amplio.
\end{abstract}

Palabras Clave: mampostería confinada; resistencia a corte; momento; interacción

\begin{abstract}
It is the contention in this study that the presence of flexural moment on confined masonry walls has an effect in the shear force that produces the first diagonal cracks due to tension. A hypothesis that relates distortion with cracking was proposed a priori and based on it a mathematical expression was developed that predicts the cracking shear force in a wall when a flexural moment is applied on its top. Afterwards, the results of a pilot experimental study consisting of two full scale confined masonry walls is presented: one tested with only lateral and axial loads, used as reference and a second one tested with flexural moment, axial force and increasing shear force. It was found, in the latter case, a reduction of the diagonal tension cracking shear load in good agreement with the prediction of the proposed mathematical model. Some observations and recommendations for an ampler study are given.
\end{abstract}

Key Words: confined masonry; shear strength; moment; interaction

Artículo recibido el 26 de febrero de 2012 y aceptado para publicación el 13 de agosto de 2012

(1) Investigador del Instituto de Ingeniería de la UNAM, en la coordinación de mecánica aplicada, jipge@pumas.iingen.unam.mx

(2) Estudiante de doctorado del Instituto de Ingeniería AManzanoT@iingen.unam.mx 


\section{INTRODUCCIÓN}

México está en el proceso de cambiar el modelo de crecimiento de sus ciudades de uno horizontal con unidades unifamiliares de vivienda a otro predominantemente vertical multifamiliar. Este cambio tiene ventajas desde el punto de vista económico y social, que pueden resumirse en que el nuevo esquema vertical es más sustentable desde el punto de vista económico y mejora la calidad de vida de sus habitantes (SEDESOL 2010).

Este cambio implica, en un futuro próximo, la construcción de un número cada vez mayor de edificios para vivienda de mayor altura, por lo que todos los aspectos relacionados con el diseño de estas estructuras y el de su entorno deben revisarse para generar las mejores soluciones: económicas y funcionales, que permitan una vida digna y segura de la población.

La mampostería conocida en la literatura como reforzada, se utiliza en países desarrollados como Japón, Estados Unidos y Nueva Zelanda y algunos otros países como Colombia, como sistema estructural de edificios de varios niveles. Por ejemplo el código de construcción en Colombia permite estructuras hasta de $50 \mathrm{~m}$ de altura aun en zonas de alta sismicidad (aproximadamente 18 niveles). Dicha mampostería consiste de piezas huecas, unidas con mortero; en las celdas de las piezas se coloca refuerzo vertical, para resistir fuerzas de flexión principalmente, y se rellenan posteriormente con concreto. En las juntas horizontales también se coloca refuerzo, esta vez para resistir fuerzas laterales. El resultado es un elemento estructural que ha sido utilizado exitosamente como elemento resistente en estructuras sometidas a la acción sísmica. Si bien este sistema se ha utilizado con éxito, es costoso.

En contrapartida el sistema de mampostería confinada, se limita en algunos países como Colombia a estructuras con uno o dos niveles. Los muros de mampostería confinada se caracterizan por su sistema constructivo: primero se construye el muro, normalmente de piezas de barro o arena cemento unidas con mortero y posteriormente se colocan elementos de borde tanto vertical como horizontal de concreto reforzado, castillos y dalas respectivamente, que confinan al muro. Los castillos y el refuerzo en ellos son tomados en cuenta para resistir flexión y carga axial. Puede además incluirse refuerzo horizontal en las juntas para resistir fuerzas laterales y mejorar notablemente su capacidad de deformación. Algunas variantes como el caso de castillos embebidos en piezas de mampostería huecas se han utilizado también. Las características de este tipo de mampostería en cuanto a resistencia a flexocompresión y cortante así como de su capacidad de deformación se ha demostrado por medio de un extenso número de pruebas de laboratorio (R. Meli 1973)(Alcocer and Meli 1995). Este tipo de mampostería representa una alternativa más económica para la construcción de edificios altos y es viable su uso si se hacen las debidas adaptaciones en cuanto al tamaño de las piezas y sus propiedades, así como del mortero y aseguramiento de la calidad durante la construcción (López O. 2011), (Cervantes Ruiz y Jean Perilliat 2009).

En México, es tradicional el considerar que las estructuras de mampostería confinada son posibles hasta cinco niveles y que después de esa altura es necesario recurrir a estructuras de acero o de concreto. El hecho de que las Normas Técnicas Complementarias para el Diseño y Construcción de Estructuras de Mampostería del Gobierno del Distrito Federal (NTCM 2004) permitan el uso del método simplificado de análisis para estructuras hasta $13 \mathrm{~m}$ de altura es, sin duda, uno de los factores que han contribuido a esta idea, aunque este requerimiento sea debido a limitaciones del método de análisis. Sin embargo, la hipótesis de estructura de poca altura sí se utiliza, aunque no de forma explícita, en otros aspectos de las normas. Esta suposición está implícita en la fórmula para determinar la resistencia a corte de los muros. Si se desea utilizar mampostería confinada para estructuras más altas, será necesario profundizar en el comportamiento de este sistema ante acciones más generales, que incluyan la combinación de fuerza axial cortante y momento. 
Uno de los efectos que potencialmente cobran importancia a medida que crece el número de niveles de una edificación es el efecto de momento flexionante sobre la resistencia a corte por deslizamiento (ver (EC6 2002) sec 6.2). Este efecto se presenta cuando un muro está sometido a fuerzas de flexión que producen que parte de la sección transversal del muro quede en tensión; dicha parte de la sección no puede contribuir a la resistencia a corte por deslizamiento.

En este artículo se propone que el momento flexionante también tiene un efecto en la carga lateral que produce el agrietamiento por tensión diagonal. Se propone a priori una hipótesis que relaciona la distorsión lateral con el agrietamiento y con base en ella se desarrolla una expresión matemática que predice la fuerza lateral que produce el agrietamiento por tensión diagonal en la presencia de un momento flexionante. Se describe el programa experimental piloto y con los resultados se verifican la validez de la hipótesis que se formuló y las predicciones obtenidas con el modelo matemático desarrollado.

\section{ANTECEDENTES}

En las NTCM se especifican expresiones para calcular la resistencia a flexión y la resistencia a cortante al primer agrietamiento, esta última se muestra en la ec. 1, ambas dependen del nivel de carga axial pero son independientes entre si.

$V_{n}(P)=0.5 v_{m} A+0.3 P \leq 1.5 v_{m} A$

En la expresión (1) $V_{n}$ es el cortante nominal resistente sin considerar el factor de reducción de resistencia, $v_{m}$ la resistencia a cortante de la mampostería, $A$ el área de la sección transversal del muro y $P$ la carga axial de compresión en el muro de la combinación de carga en estudio. Se utilizará aquí $v_{m}$ para hacer referencia al valor medio del esfuerzo cortante resistente de pruebas de compresión diagonal de probetas tomadas durante la construcción del muro, para distinguirlo de $v^{*}$ que es un valor de diseño. La ecuación (1) fue calibrada con pruebas de laboratorio en las que se ensayaron muros con relación de aspecto, altura entre longitud del muro $w=H / L \approx 1$, sometidos a carga gravitacional y lateral. En dichas pruebas el momento en la base del muro es $M_{b}=V H$, de manera que los coeficientes en la ec. 1 ya toman en cuenta el efecto de ese momento; sin embargo, ni el experimento ni la expresión contemplan el efecto de un momento aplicado en el extremo superior del muro.

Algunos autores (R. Meli 1975), (Alvarez 1996), (Zeballos, y otros 1992), han hecho referencia a la relación de aspecto y su efecto en la resistencia a cortante, que bien puede interpretarse como referencia al problema de interacción cortante-momento o al efecto del momento en la resistencia a corte. En los trabajos mencionados, la relación de aspecto se refiere, en ocasiones, a la razón entre la altura del muro y su longitud $(H / L)$ y en otras ocasiones a la relación de aspecto de cortante, definida como el momento flexionante dividido entre el producto del cortante y la longitud del muro $(M / V L)$, que es a su vez una medida de la cantidad de momento en el muro. Para el caso de un muro de un nivel, ambos conceptos están ligados pues el momento en la base vale $M=V H$ y por tanto la relación $M / V L$ es igual a $H / L$, sin embargo en el caso de muros de varios niveles, el momento $M=M_{a}+V H$ donde $M_{a}$ es el momento en el extremo superior del muro y $H$ es la altura de entrepiso, y por lo tanto $M / V L$ ya no corresponde a la relación de aspecto. El momento $M_{a}$ normalmente crece con el número d niveles.

Si el muro tiene alguna restricción al giro, $M_{a}<0$. Esto es, el momento en el extremo superior del muro va en dirección opuesta al generado por el cortante; si el giro está totalmente restringido, el momento $M_{a}=-V H / 2$ y el momento en la base del muro decrece hasta llegar a ser de la mitad del que tendría si estuviera en voladizo $(M=V H / 2)$; en ese caso $M / V L=H / 2 L$ por lo que se considera que la 
esbeltez se reduce a la mitad. En ambos casos puede interpretarse a la cantidad $H_{e}=M / V$ como una altura efectiva y a la relación $H_{e} / L$ como una relación de aspecto efectiva. En la literatura no es consistente la referencia a la altura del muro en $H / L$ : en ocasiones es la altura del muro hasta la azotea (Zeballos, y otros 1992), en otras se refieren a la altura efectiva $H_{e}$ y en otras a la altura de entrepiso, se usará aquí la última de ellas. Contrario a lo que en la literatura suele expresarse como un efecto de la "esbeltez" del muro, la relación $M / V L$ puede representar la cantidad de momento en el muro e indirectamente la condición de frontera, mas no los efectos de la relación de aspecto $H / L$ propiamente dichos, como son el cambio de la distribución de los esfuerzos cortantes y normales en la sección de los muros largos, o la proporción de los desplazamientos debidos a flexión y cortante (R. Meli 1975).

En varios códigos como el Americano, el de Nueva Zelanda etc. ((Davis 2008)) se utiliza el parámetro $M V / L$ para incrementar la resistencia básica de la mampostería de muros largos. Este incremento de la resistencia a medida que se reduce la relación de aspecto la ha reportado (Voon y Ingham 2006) y (Alvarez 1996), sin que existiera alguna explicación teórica hasta recientemente (Perez Gavilan, Flores y Alcocer 2012). En la fig. 1 se muestra la variación de la resistencia al agrietamiento normalizada con respecto a la resistencia nominal de las NTCM de siete muros de mampostería confinada a escala natural (ME1 a ME7) con relaciones de aspecto $(H / L=2.13,1.48,1.18,0.96,0.58,0.40,0.27$ ) respectivamente (Pérez-Gávilan, y otros 2010). Los muros fueron probados en México usando el protocolo de prueba descrito en las NTCM para un nivel de carga axial de $5 \mathrm{~kg} / \mathrm{cm}^{2}$. Se observa que la resistencia al agrietamiento por tensión diagonal, aumenta al disminuir la relación de aspecto. Es importante notar que para los muros esbeltos (M3 a M1) la resistencia no se reduce como podría esperarse si se siguiera la tendencia de los muros ME7 a ME4. Esta observación será de importancia cuando se comparen las predicciones de la resistencia al agrietamiento hechas aquí con las propuestas en el código peruano. Las fórmulas en los códigos mencionados son aplicables si $M / V L<1$. Para entender los casos que dicha limitación implica, supóngase que $M_{a}=0$, entonces $M / V L<1$ solo si $H / L<1$, esto es, aplica para muros largos en voladizo. Para muros esbeltos $H / L>1$ las fórmulas son aplicables si $M_{a}<$ 0 , que implica que hay una restricción al giro que reduce la esbeltez efectiva del muro. $M_{a}$ puede ser $>0$ solo en el caso de muros largos $(L>H)$. En cualquier caso el momento en el extremo superior del muro está limitado a $M_{a}<V(L-H)$. Se supone aquí que $V>0$. En conclusión, las fórmulas de diseño mencionadas no consideran todas las combinaciones de momento $M_{a}$ y relación de aspecto $H / L$, en particular muros esbeltos con momento $M_{a}>0$.

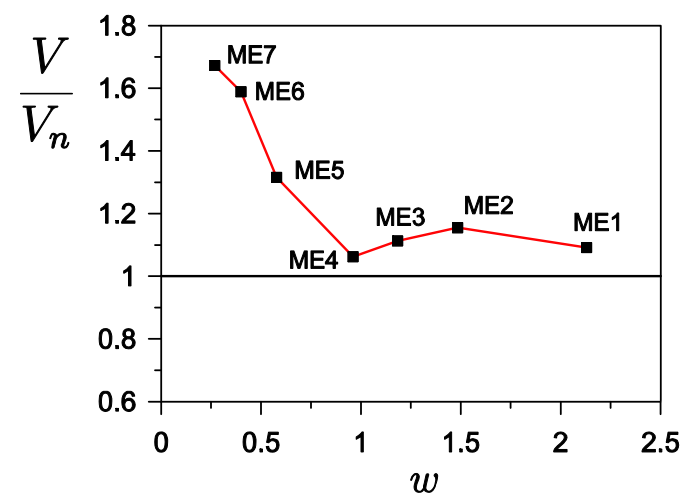

Figura 1. Resistencia al agrietamiento por tensión diagonal y la relación de aspecto.

En muros esbeltos a pesar de que se ha observado una reducción de la resistencia a cortante (Matsumura 1988), (Zeballos, y otros 1992) esta no ha sido incluida en los códigos a excepción del caso particular del reglamento de Perú. En la ec. 2 se presenta la expresión utilizada por el código peruano para 
establecer la resistencia nominal a corte por tensión diagonal. En la fig. 2.b se presenta la variación de la resistencia básica de la mampostería con la relación $(M / V L)$.

$$
V_{n}=0.5 \alpha v_{m} A+0.23 P \quad \alpha=\left(\frac{V L}{M}\right) \quad \frac{1}{3}<\alpha<1
$$

En el trabajo que dio lugar a dicha especificación (Zeballos, y otros 1992) el efecto que se estudia es la modificación de los esfuerzos principales a tensión que produce el agrietamiento del muro. Dicho cambio en los esfuerzos principales se observa en muros con distinta relación de aspecto y un creciente número de niveles de la estructura. (fig 2.a). La interpretación
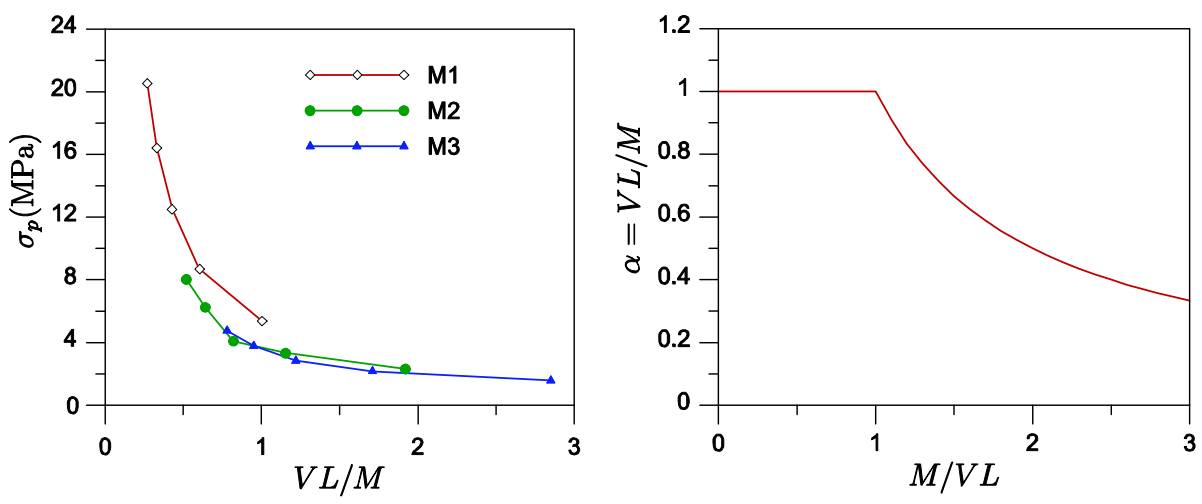

Figura 2. a) Esfuerzo máximo de tensión para muros con uno dos y tres paneles, M1, M2 y M3 respectivamente de uno a cinco niveles (15 muros en total) y b) factor $\alpha$ del código peruano.

que aquí se hace de los resultados de ese estudio difiere de la de los autores en que parte del efecto observado en la resistencia a tensión diagonal se atribuye a la presencia de momento y no solo a la relación de aspecto. En dicho trabajo no es posible distinguir entre ambos efectos. En (Pérez-Gavilán y Manzano 2012) se hace una extensa reflexión de dicho estudio.

\section{HIPÓTESIS}

Para poder predecir la fuerza lateral que produce el agrietamiento, se hace una hipótesis que relaciona el desplazamiento lateral con el agrietamiento. La hipótesis consiste en establecer que el agrietamiento por tensión diagonal se produce para un desplazamiento relativo del muro, independientemente de que dicho desplazamiento sea producido por una fuerza lateral solamente, como lo asumen las NTCM, o por la combinación de una fuerza lateral y un momento $M_{a}$ en el extremo superior del muro. Haciendo uso de esta hipótesis, se deduce que la distorsión lateral producida por la fuerza cortante más la producida por el momento flexionante debe ser igual a la que se produce por la fuerza cortante que predicen las NTCM (fig. 3).

Sea $\delta_{0}$ el desplazamiento debido a la fuerza cortante $\left(V_{n}\right)$ que produce el agrietamiento cuando no hay momento. Ese mismo desplazamiento producirá el agrietamiento si una parte de dicho desplazamiento $\delta_{V}$ es debida una fuerza cortante $V_{n}^{\prime}$ y la parte restante $\delta_{M}$ es producida por un momento en el extremo superior del muro $M_{a}$.

$\delta_{V}+\delta_{M}=\delta_{0}$

expresando la ec. 3 en términos de cortante se tiene 
$V_{n}^{\prime}=V_{n}-\delta_{M} K_{0}$

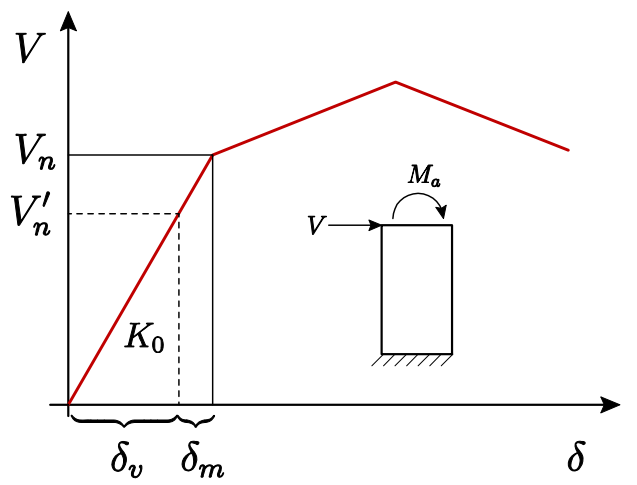

Figura 3. Modelo de resistencia cortante al primer agrietamiento

donde

$\delta_{M}=\frac{M_{a} H^{2}}{2 E I}, \quad K_{0}=\frac{k_{f} k_{v}}{k_{f}+k_{v}}, \quad k_{f}=\frac{3 E I}{H^{3}}, \quad k_{v}=\frac{G A}{\kappa H}, \quad I=\frac{t L^{3}}{12}, \quad A=t L$

En las expresiones anteriores $E$ es el módulo de elasticidad, $G$ el módulo de cortante, $I$ el momento de inercia, $A$ el área de la sección transversal del muro, $\kappa$ el factor de forma para obtener el área de cortante, $t$ el espesor del muro y $H$ la altura del muro referida al centro de la losa.

Sustituyendo los términos de la ec. 5 en la ec. 4, puede calcularse el valor de la resistencia a corte en función del momento $M_{a}$ y del cortante nominal, como:

$V_{n}^{\prime}\left(P, M_{a}\right)=V_{n}(P)-\frac{M_{a} H^{2}}{2 E I} K_{0} ; \quad V_{n}^{\prime}\left(P, M_{a}\right)=V_{n}(P)-\frac{3}{2} \frac{M_{a}}{H} \frac{k_{v}}{k_{f}+k_{v}}$

De la ec. 6 se observa que para muros muy largos el cortante resistente es igual al nominal, ya que los desplazamientos debidos a flexión son muy pequeños $\left(k_{f} \rightarrow \infty\right)$. Para muros muy esbeltos $\left(k_{v} \gg k_{f}\right)$ la reducción es asintótica a $3 M_{a} / 2 H$, que corresponde a la máxima reducción posible de la fuerza cortante para un valor de momento dado. El momento $M_{a}$ puede considerarse menor a cero si va en sentido opuesto al que genera el cortante, lo que ocasiona un aumento de la resistencia a corte. Agrupando los valores que solamente dependen de la geometría del muro, la ec. 6, puede rescribirse como

$V_{n}^{\prime}=V_{n}-\frac{M_{a}}{H_{k}}, \quad H_{k}=\frac{2}{3} \frac{k_{f}+k_{v}}{k_{v}} H$

\section{ESTIMACIÓN DE LA REDUCCIÓN DE LA RESISTENCIA A CORTE}

Para estimar la reducción de la resistencia a cortante usando la hipótesis de la sección anterior se introduce el cociente $\alpha=V_{n}^{\prime} / V_{n}$. Utilizando los parámetros $w=H / L, M_{a}=\beta V_{n}^{\prime} H / 2$ y $\eta=G / E$ y sustituyendo la ec. 5 en la ec. 4 se obtiene 
$\alpha=\frac{1}{1+\frac{15 \beta \eta w^{2}}{20 \eta w^{2}+6}}$

Conviene expresar el momento en el extremo superior del muro como $M_{a}=\beta V_{n}^{\prime} H / 2$ ya que $\beta$ controla la condición de frontera. Si $\beta=-1$ el muro tiene restringido el giro de su extremo superior, si $\beta=0$ el valor de $M_{a}=0$ y el muro esta en voladizo $(\alpha=1)$, finalmente si $\beta>0$ el momento en el extremo superior $M_{a}>0$ y el cociente $\alpha<1$ lo que indica una reducción de la resistencia a cortante. En la fig. 4.a se muestra el valor de $\alpha$ para distintos valores de momento en función de la relación de aspecto y de las propiedades elásticas de la mampostería. En el análisis se han considerado dos valores de $\eta$, en línea continua $\eta=0.4$ y en línea discontinua $\eta=0.2$. El primero de estos valores es el que especifican las NTCM, mientras que el segundo es el valor medio del rango $[0.1,0.3]$ de $\eta$ reportado por varios investigadores (Bazan 1980), (Pérez-Gávilan, y otros 2010). Se observa que la reducción de la resistencia a corte es menor a medida que la relación $\eta=G / E$ de la mampostería es menor, debido a que la deformación por cortante es mayor. El valor de $\alpha$ se hace asintótico para muros muy esbeltos $w \rightarrow \infty$

$\lim _{w \rightarrow \infty} \alpha=\frac{4}{4+3 \beta}$

que es consistente con $V_{n}^{\prime}=V_{n}-1.5 M_{a} / H$ obtenido anteriormente. Cuando los muros son muy largos $(w \rightarrow 0$ ) el cociente vale $\alpha=1$, pues la deformación por flexión es tan pequeña que es como si estuviera en voladizo.

En la fig. 4.b se presenta la reducción de la fuerza cortante en función del momento $M_{a}$ para distintas relaciones de aspecto $w$ y propiedades elásticas. Las curvas tienden a cero al incrementarse $\beta$, sin embargo, la velocidad con que decrece $\alpha$ va reduciéndose al incrementarse el momento, particularmente en los muros esbeltos con relaciones de aspecto $w>1$.
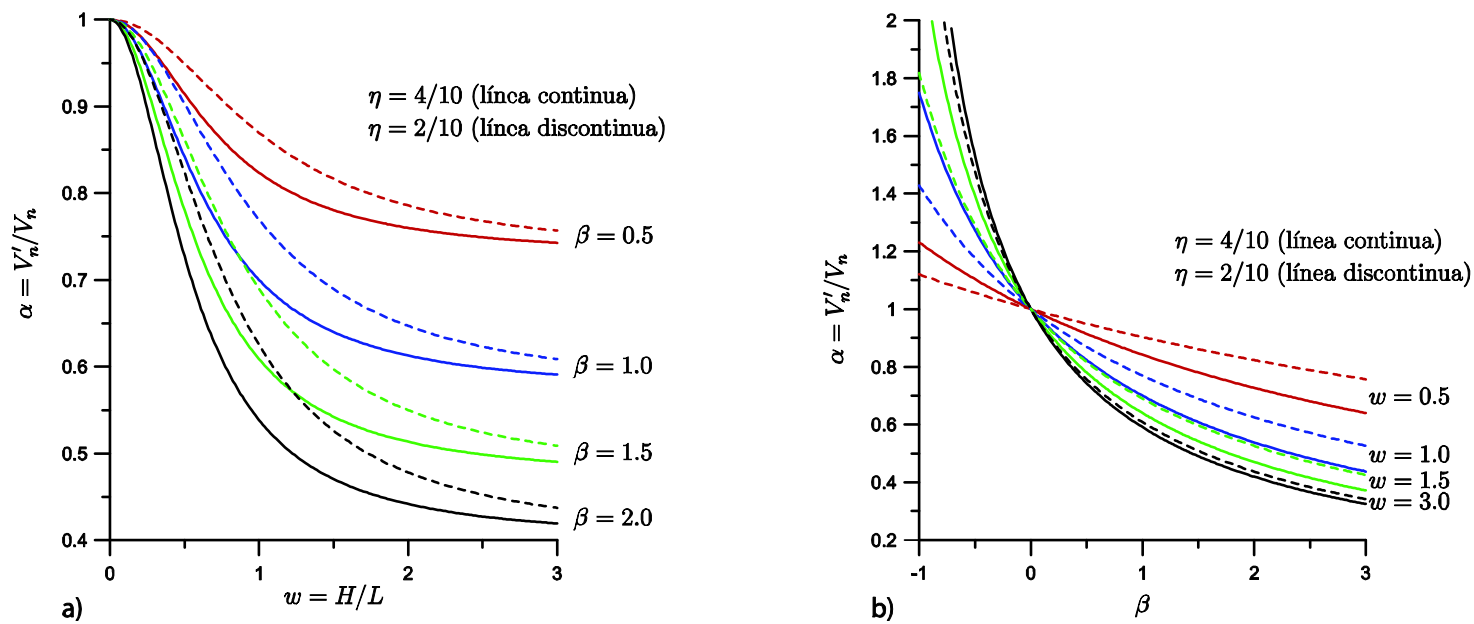

Figura 4. Relación de cortantes para muro en voladizo para distintos valores de $w, M_{a}$ y $\eta$

\section{DIAGRAMA DE INTERACCIÓN MODIFICADO}

Con la finalidad de visualizar la respuesta de los muros a cortante en presencia del momento flexionante actuando de manera simultánea, se construyeron diagramas de interacción de acuerdo a las 
hipótesis de sección plana contenida en las NTCM. Sobre estos se dibujaron nuevos diagramas que también dependen de la carga axial pero ahora los momentos son los producidos por el cortante nominal más el momento en el extremo superior del muro. Estos nuevos diagramas representan la falla por cortante, sin considerar ningún tipo de refuerzo horizontal en el muro.

Los puntos sobre el diagrama de interacción del muro $\left(M_{e}, P_{e}\right)$ provocan la falla a flexocompresión en el muro, sin embargo, la falla en el muro puede presentarse antes por cortante, por tanto hay que evaluar el cortante resistente nominal $V_{n}\left(P_{e}\right)$, para el nivel de carga axial $P_{e}$ y el momento máximo que podría producirse en el instante en que el muro falla por corte, esto es

$M_{v}\left(P, M_{a}\right)=V_{n}(P) H+M_{a}$

Si este momento es menor al obtenido para el diagrama de interacción $M_{e}$ entonces el momento máximo debe limitarse a $M_{v}$. El punto $\left(M_{v}, P_{e}\right)$ es el punto del diagrama de interacción modificado por cortante, para un valor específico de $M_{a}$. El diagrama de interacción modificado se construye uniendo con una curva los puntos calculados. Si se utiliza $V_{n}^{\prime}$ en vez de $V_{n}$ en la ec. 10, se tendrá un punto del diagrama de interacción modificado incluyendo el efecto del momento $M_{a}$ en la resistencia a corte.

En la fig. 5 se presenta el diagrama de interacción y el diagrama de interacción modificado para distintos niveles de momento $M_{a}$, junto con los diagramas modificados considerando la reducción de la resistencia a corte (líneas punteadas). En el diagrama se observa una rama ascendente que varía con la carga axial hasta un cierto valor $P_{m}$, después del cual, la carga axial ya no influye en el valor del momento máximo en el diagrama modificado,. Esto se debe a que la resistencia máxima a cortante establecida en las NTCM debe ser menor o igual a $1.5 v_{m} A$. El valor de $P_{m}$ se obtiene de la ecuación

$0.5 v_{m} A+0.3 P_{m}=1.5 v_{m} A$

de donde

$P_{m}=\frac{v_{m} A}{0.3}$

Posteriormente, el diagrama modificado tiene una rama vertical que corresponde al momento $M_{v}=V_{n} H+$ $M_{a}$ de acuerdo a las normas vigentes (líneas continuas) y $M_{v}=V_{n}^{\prime} H+M_{a}$ cuando se utiliza el cortante reducido por momento flexionante (líneas discontinuas). Eventualmente $M_{v}$ será mayor que $M_{e}$ en cuyo caso se limita a $M_{v}=M_{e}$. En la fig. 5 la curva [1] representa el diagrama de interacción a flexocompresión, la [2] el diagrama de interacción modificado por cortante cuando $M_{a}=0$, la curva [3] es el diagrama de interacción modificado por cortante para $M_{a}=176.52 \mathrm{kN}-\mathrm{m}$, se indica considerando que la resistencia a corte no se ve afectada por el momento: $M_{v}=V_{n}(P) H+M_{a}$, la curva [4] es el diagrama de interacción modificado por cortante afectado por momento: $M_{v}=V_{n}^{\prime}\left(P, M_{a}\right) H+M_{a}$. La zona [5] indica la ocurrencia de falla a flexocompresión $M_{e}<M_{v}\left(P, M_{a}\right)$ del muro con excentricidades $M_{b} / P$ indicadas, y finalmente la zona [6] de falla a flexocompresión solo para el caso en que el que $M_{a}=176.52 \mathrm{kN}-\mathrm{m}$ o mayor. $P_{m}$ es el valor de carga axial a partir de la cual la resistencia a corte ya no aumenta. 


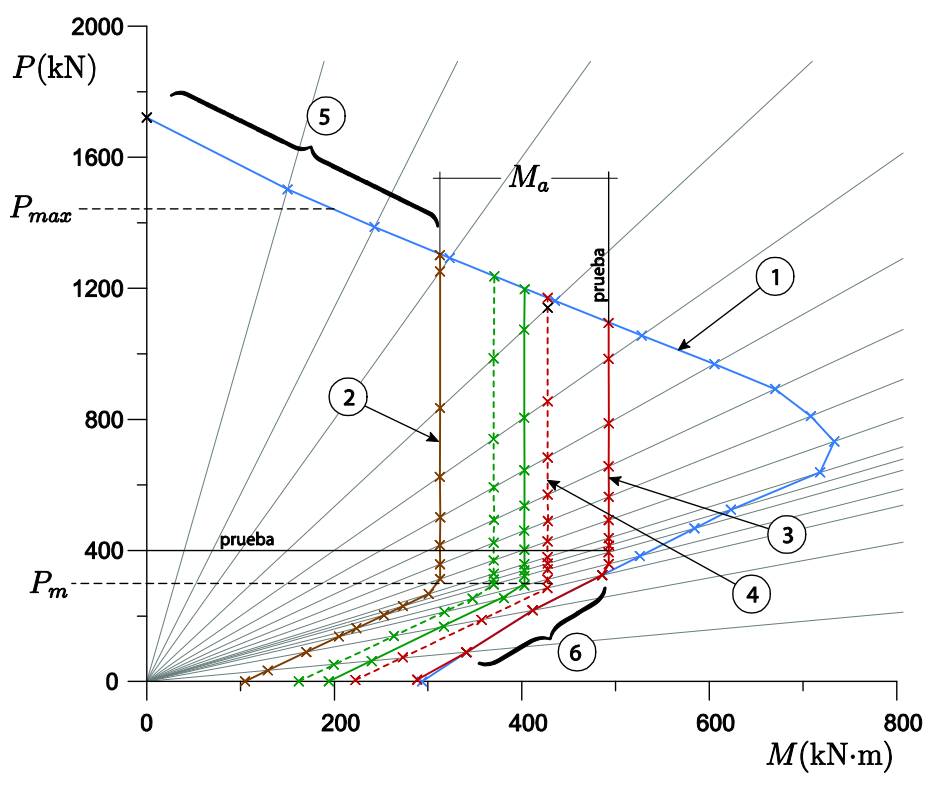

Figura 5. Diagrama de interacción modificado

\section{PROGRAMA EXPERIMENTAL}

\section{Descripción de los especímenes}

Se construyeron dos especímenes de prueba M1 y M2 con relación de aspecto $w=1$. El muro M1 se usó de referencia y se probó sin momento flexionante. Los muros fueron construidos por el mismo maestro albañil, con procedimientos constructivos idénticos y con materiales tomados de un mismo lote.

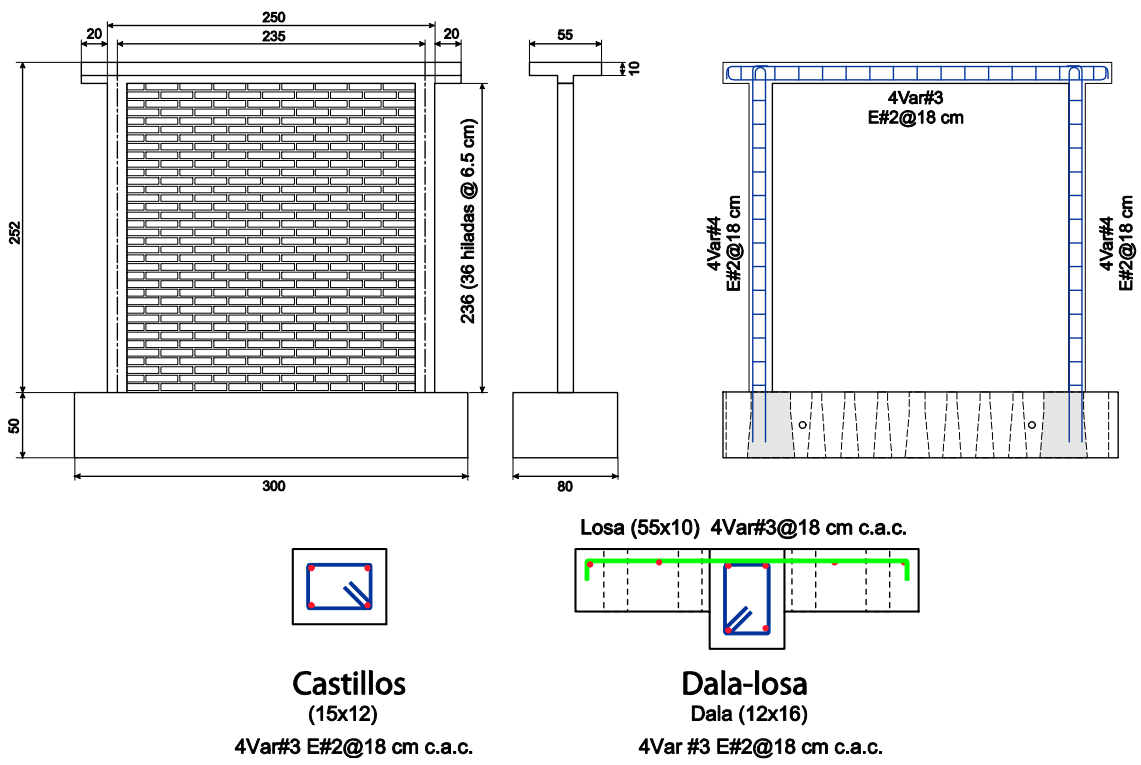

Figura 6. Geometría nominal, armado de los especímenes y geometría de elementos de concreto 
Tabla 1. Propiedades índice experimentales de cada espécimen

\begin{tabular}{ccccccccc}
\hline & $\begin{array}{c}f_{m} \\
(\mathrm{MPa})\end{array}$ & $\begin{array}{c}v_{m} \\
(\mathrm{MPa})\end{array}$ & $\begin{array}{c}E_{m} \\
(\mathrm{MPa})\end{array}$ & $\begin{array}{c}G_{m} \\
(\mathrm{MPa})\end{array}$ & $\begin{array}{c}f^{\prime}{ }_{c} \\
(\mathrm{MPa})\end{array}$ & $\begin{array}{c}E_{c} \\
(\mathrm{MPa})\end{array}$ & $\begin{array}{c}f_{y} \\
(\mathrm{MPa})\end{array}$ & $\begin{array}{c}E_{s} \\
(\mathrm{MPa})\end{array}$ \\
\hline $\mathrm{M} 1$ & 4.45 & 0.31 & 623.11 & 171.52 & 23.24 & 10352.68 & 404.13 & 205998 \\
$\mathrm{M} 2$ & 4.53 & 0.33 & 785.51 & 139.65 & 21.28 & 8088.33 & 404.13 & 205998 \\
$\mathrm{Cv}(+)$ & 0.169 & 0.266 & 0.249 & 0.088 & 0.022 & 0.019 & - & - \\
\hline
\end{tabular}

(+) Promedio de los coeficientes de variación del muro M1 y M2

Las dimensiones nominales de los especímenes fueron $250 \mathrm{~cm}$ de ancho por $247 \mathrm{~cm}$ de alto y se construyeron con piezas de tabique de barro rojo macizo tipo artesanal de $23.4 \times 11.8 \times 5.3 \mathrm{~cm}$ de sección unidos con mortero tipo I en proporción cemento, cal, arena 1:1/2:3. Los castillos se armaron con cuatro barras Grado 42 de $9.5 \mathrm{~mm}$ de diámetro (No. 3) y el refuerzo transversal se realizó con estribos de alambrón de $6.35 \mathrm{~mm}$ de diámetro (No. 2) espaciados a cada $18 \mathrm{~cm}$ cumpliendo con las disposiciones de las NTCM. La sección transversal de las dalas fue de $12 \times 16 \mathrm{~cm}$ y se reforzaron con cuatro varillas Grado 42 de 9.5 mm de diámetro (No. 3) y estribos de alambrón de $6.35 \mathrm{~mm}$ de diámetro (No. 2). Todo el refuerzo longitudinal de dalas y castillos se remató con ganchos a $90^{\circ}$ y $180^{\circ}$ (fig. 6).

Las propiedades de los materiales se determinaron experimentalmente para cada muro de acuerdo con los procedimientos normativos correspondientes; la tabla 1 contiene el resumen de propiedades. Se hicieron dos pilas de ocho piezas sobrepuestas en concordancia con las NTCDF-2004, dos muretes por muro, pilas y muretes probados de acuerdo a lo establecido en el anteproyecto de norma APROY-NMX000-ONNCCE-2009 y tres cilindros de concreto de los castillos, probados de acuerdo la norma NMX-C414-ONNCCE-2004, NMX-C-083-ONNCCE-2002, NMX-C-109-ONNCCE-2004 y NMX-C-128-1997ONNCCE.

\section{Sistema de carga}

El esquema completo del sistema para la aplicación de cargas se muestra en la fig. 7. La carga lateral se aplicó mediante un gato hidráulico con capacidad de 980 kN y $350 \mathrm{~mm}$ de carrera anclado en un extremo al muro de reacción y en el otro al extremo de una viga de acero, sujeta a todo lo largo del muro para transmitir la carga de manera uniforme y a la altura de la losa simulando la forma en que las fuerzas de inercia se transmiten al muro durante un sismo. La carga vertical y las componentes del momento flexionante se aplicaron mediante dos actuadores verticales con capacidad de $490 \mathrm{kN}$ y $350 \mathrm{~mm}$ de carrera colocados a cada lado del muro. Las celdas de carga de los actuadores corresponden con la capacidad de los actuadores $100 \mathrm{kN}$ y $500 \mathrm{kN}$ para el actuador horizontal y actuadores verticales respectivamente. Dada la rigidez de la viga se consideró que la fuerza axial es uniforme en el muro; sin embargo, simulaciones realizadas mediante un modelo de elementos finitos mostraron que los esfuerzos axiales son uniformes solo en la mitad baja del muro y que existen concentraciones de esfuerzos en las esquinas que forman los castillos con la dala; no obstante no se observó algún efecto por este hecho.

\section{Instrumentación externa}

Consistió en la colocación de transductores de desplazamiento lineal de 25, 50 y $100 \mathrm{~mm}$ de capacidad de medición dispuestos en la parte posterior y lateral del muro tal y como se muestra en la fig. 8.

\section{Instrumentación interna}

Esta consistió en la colocación de seis galgas extensométricas (“strain gages”) Tokyo Sokki Kenkyujo modelo FLA-5-11-5L, tres por castillo, dos adheridos a las barras de refuerzo longitudinales de 
los castillos en su parte inferior y uno al segundo estribo de alambrón, todos en la parte posterior del muro como se muestra en la fig. 9.

\section{Adquisición de datos}

Los sistemas de adquisición de datos empleados para la captura fueron:

1. Controlador MTS FlexTest 60 provisto con software Station Manager y

2. Sistema de adquisición de datos TDS-300 Tokyo Sokki Kenkyujo

Dado que el sistema de adquisición de datos no está sincronizado con el controlador MTS y que la frecuencia de captura es diferente, las señales deben ser alineadas, filtradas, y decimadas en un postproceso auxiliándose del programa para computadora Alinreg (Pérez Gavilán 2008).

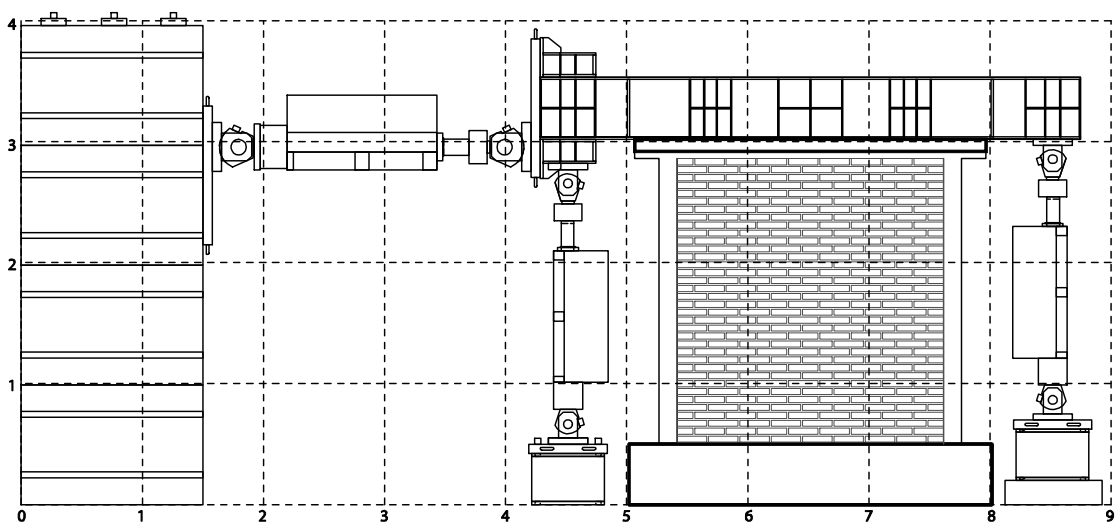

Figura 7. Vista este del sistema para la aplicación de cargas

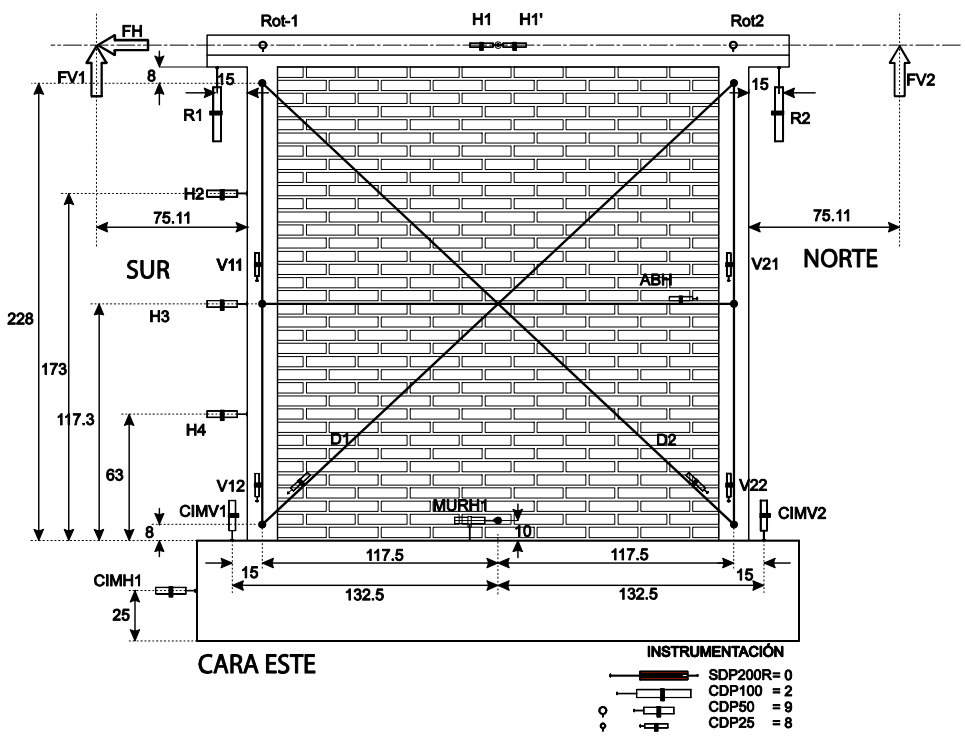

Figura 8. Arreglo general de instrumentación externa 

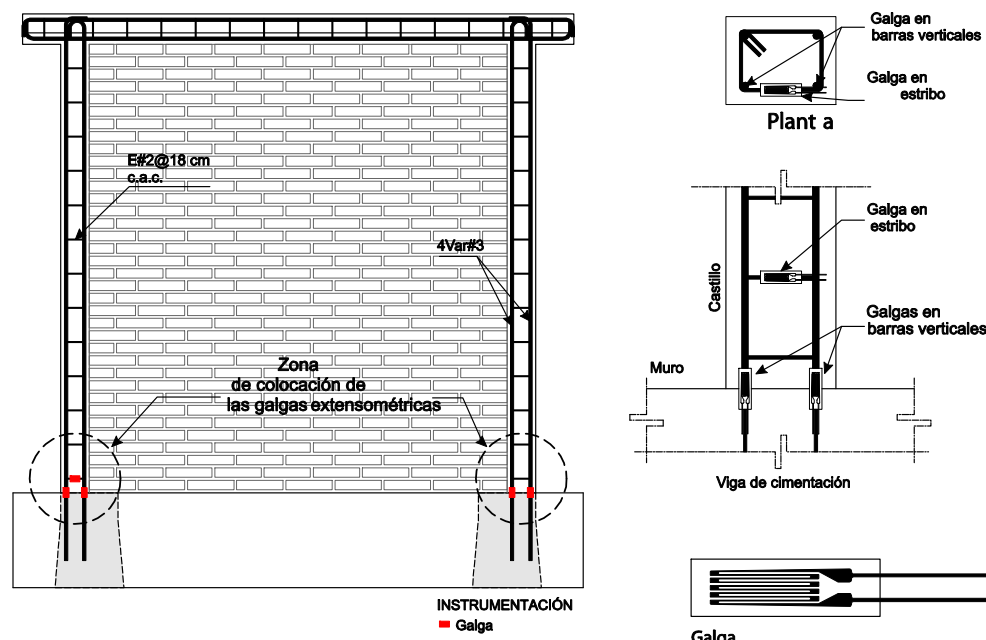

Figura 9. Posición esquemática de galgas extensométricas.

\section{Secuencia de carga}

Se seleccionó un momento $M_{a}$, de $176 \mathrm{kN} \cdot \mathrm{m}$ para el muro M2 y el nivel de carga axial para ambos muros se fijó en $P=392 \mathrm{kN}(\sigma=1.32 \mathrm{MPa})$; la carga corresponde aproximadamente a la de un muro de planta baja de un edificios de aproximadamente 10 niveles.

El protocolo de aplicación de la carga para la condición de momento $M_{a}=0$ (muro M1) fue muy similar al protocolo de las NTCM salvo que después de los ciclos por control de carga a $0.25 V_{n}$ y $0.5 V_{n}$ se procedió con los ciclos por control de desplazamiento, comenzando con una distorsión de 0.0015 , para continuar de acuerdo a lo establecido en las NTCM. Las modificaciones realizadas permitieron hacer las pruebas en forma automatizada minimizando así la posibilidad de errores durante la ejecución de la prueba (fig. 10)

En el muro M2 el momento se aplicó en forma cíclica. Durante la fase de control de carga se aplicaron los primeros dos ciclos con $0.25 V_{n}$ y $0.5 M_{a}$ y los dos siguientes con $0.5 V_{n}$ y $M_{a}$. Iniciando la fase de control de desplazamientos, se aplicaron rampas $\left(\gamma=0.003, M_{a}\right)$ y $\left(\gamma_{j}, M_{a}\right)$, es decir, el momento se incrementa linealmente hasta llegar a $M_{a}$ cuando la deformación lateral alcanza una deformación de 0.003, posteriormente se deja fijo el valor del momento y se incrementa la distorsión lateral hasta el valor programado $\gamma_{j}$. Esta forma de aplicar el momento garantiza que el momento máximo $M_{a}$ que se tenía contemplado en la prueba se alcance antes de que se presente el agrietamiento y la falla del muro.

\section{RESULTADOS}

\section{Puntos críticos y distorsiones}

En la tabla 2 se incluyen los valores de desplazamiento y fuerza cortante de los puntos críticos: el primer agrietamiento diagonal, resistencia máxima y última. Los valores de las deformaciones correspondientes a la carga de agrietamiento, a la carga máxima y última así como las ductilidades se presentan en la tabla 3. 


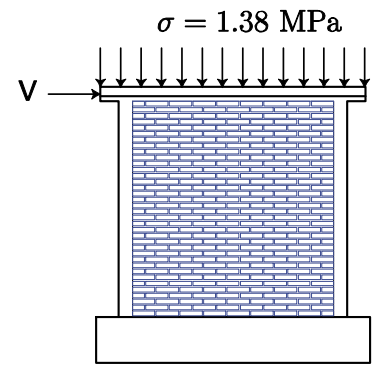

M1

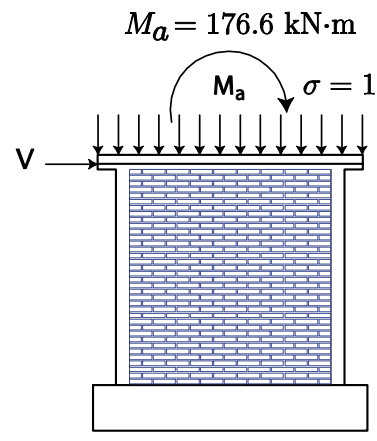

M2
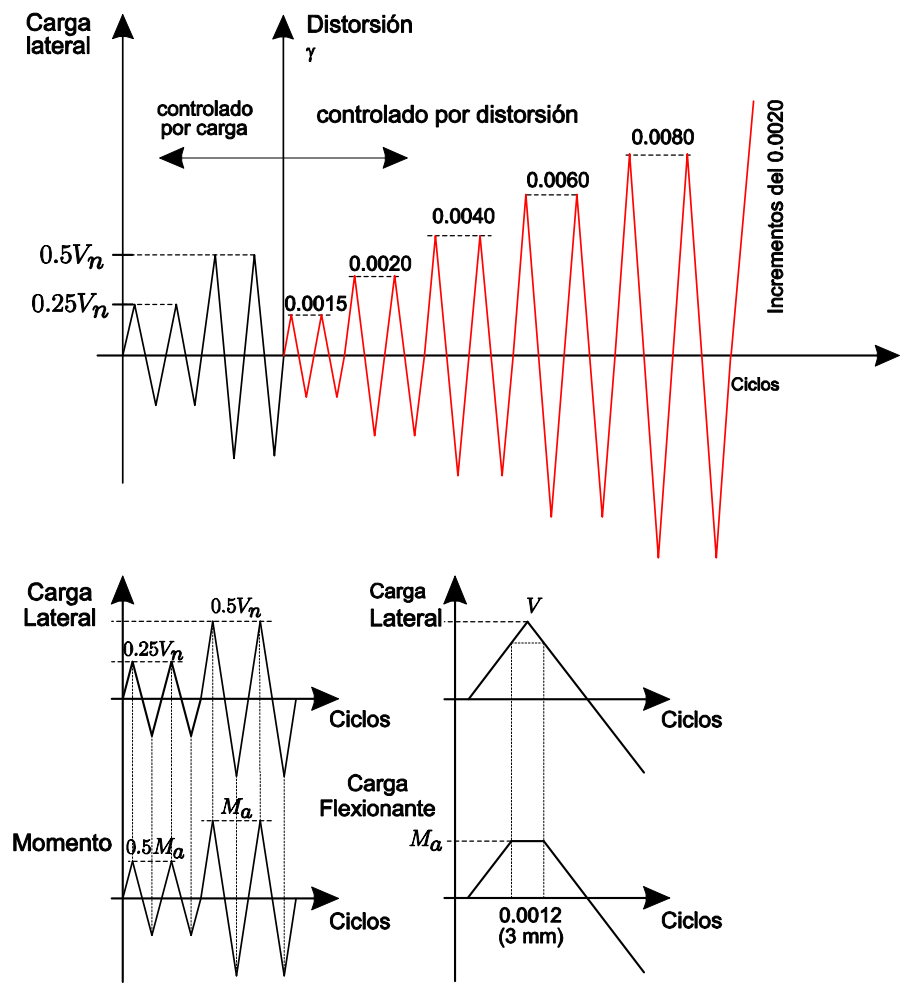

Figura 10. Secuencia de carga en muros M1 y M2

Tabla 2. Valores de los puntos críticos

\begin{tabular}{lcccccccccccc}
\hline \multicolumn{1}{c}{ Agrietamiento } & \multicolumn{1}{c}{ Máximos } & \multicolumn{4}{c}{ Últimos } \\
\hline \multirow{2}{*}{ Muro } & $\begin{array}{c}V_{c}^{+} \\
(\mathrm{kN})\end{array}$ & $\begin{array}{c}d_{c}^{+} \\
(\mathrm{mm})\end{array}$ & $\begin{array}{c}V_{c}^{-} \\
(\mathrm{kN})\end{array}$ & $\begin{array}{c}d_{c}^{-} \\
(\mathrm{mm})\end{array}$ & $\begin{aligned} V_{\max }^{+} \\
(\mathrm{kN})\end{aligned}$ & $\begin{array}{c}d_{\max }^{+} \\
(\mathrm{mm})\end{array}$ & $\begin{array}{c}V_{\max }^{-} \\
(\mathrm{kN})\end{array}$ & $\begin{array}{c}d_{\max }^{-} \\
(\mathrm{mm})\end{array}$ & $\begin{array}{c}V_{u}^{+} \\
(\mathrm{kN}\end{array}$ & $\begin{array}{c}d_{u}^{+} \\
(\mathrm{mm})\end{array}$ & $\begin{array}{c}V_{u}^{-} \\
(\mathrm{kN})\end{array}$ & $\begin{array}{c}d_{u}^{-} \\
(\mathrm{mm})\end{array}$ \\
\hline \multirow{2}{*}{ M1 } & 143.4 & 4.75 & -151.1 & -4.50 & 188.9 & 15.12 & -151.1 & -94.50 & 170.2 & 15.37 & -111.0 & -14.32 \\
M2 & 110.5 & 4.97 & -96.2 & -4.94 & 176.4 & 19.62 & -154.7 & -14.72 & 176.4 & 19.62 & -150.5 & -14.76 \\
\hline
\end{tabular}

Tabla 3. Distorsiones y ductilidades

\begin{tabular}{ccccccc}
\hline & \multicolumn{1}{c}{$\gamma_{c}$} & $\gamma_{\max }$ & $\gamma_{u}$ & $\gamma_{\max } / \gamma_{c}$ & $\gamma_{u} / \gamma_{c}$ & $\begin{array}{c}E_{d} \\
\mathrm{kN} \cdot \mathrm{mm}\end{array}$ \\
\hline M1 & 0.00193 & 0.00616 & 0.00626 & 3.19 & 3.24 & 900 \\
M2 & 0.00203 & 0.00801 & 0.00801 & 3.95 & 3.95 & 752 \\
\hline \multicolumn{2}{l}{ Ed = Energía disipada hasta el agrietamiento }
\end{tabular}

Obsérvese que las distorsiones al agrietamiento son muy similares en el muro M1 y M2, que es la hipótesis en que se basa la predicción de la disminución de la fuerza cortante por momento.

\section{Patrones de agrietamiento}

El aspecto final del agrietamiento de los muros M1 y M2 se muestra en la fig. 11. En ambos muros el agrietamiento dominante es esencialmente por cortante, sin embargo, el muro M2 exhibe un 
agrietamiento más distribuido y un mayor número de grietas de poco espesor con respecto al del muro M1. Se observó que el castillo sur del muro M2 presentó aplastamiento del recubrimiento atribuido a la flexión en el muro. La parte inferior del castillo norte de ambos muros falló primero y experimentó mayor concentración de daño que su contraparte sur.

\section{Curvas de histéresis}

Las curvas carga contra distorsión de los muros M1 y M2 se muestran en la fig. 12. Pese a que el comportamiento de los muros exhibe cierto nivel de simetría, es notorio el hecho de que la resistencia máxima es asimétrica y mayor en la rama positiva. Esta diferencia es mayor en el muro M1 y menos marcada en el muro M2.

Al observar el cambio de pendiente entre ciclo y ciclo se deduce que el daño ocurre en forma más abrupta en el muro M1 mientras que en el muro M2 el daño se presenta en forma gradual. Resulta claro que en el muro M2 los ciclos son más uniformes y amplios y con cambios menos drásticos entre ciclo y ciclo. El comportamiento del muro M2 parece, en general, bastante estable.
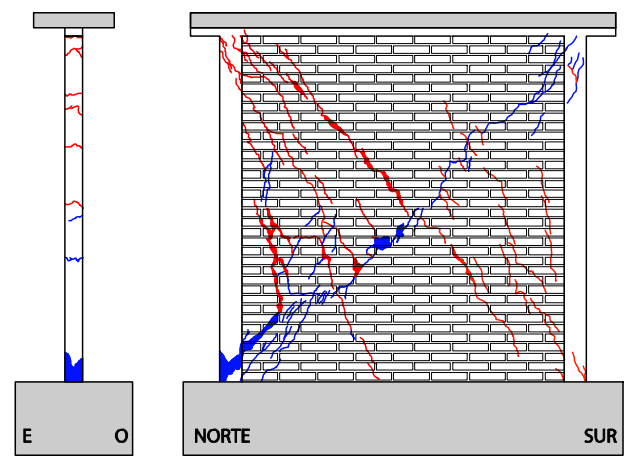

M1

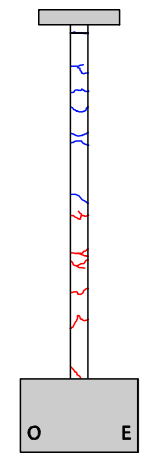

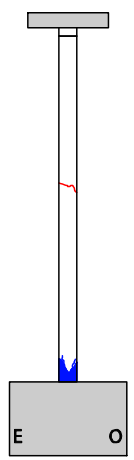

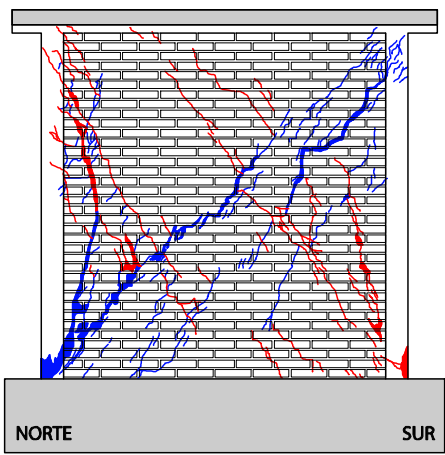

M2

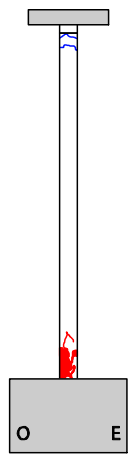

$0 \quad \mathrm{E}$

Figura 11. Aspecto final del agrietamiento de los muros

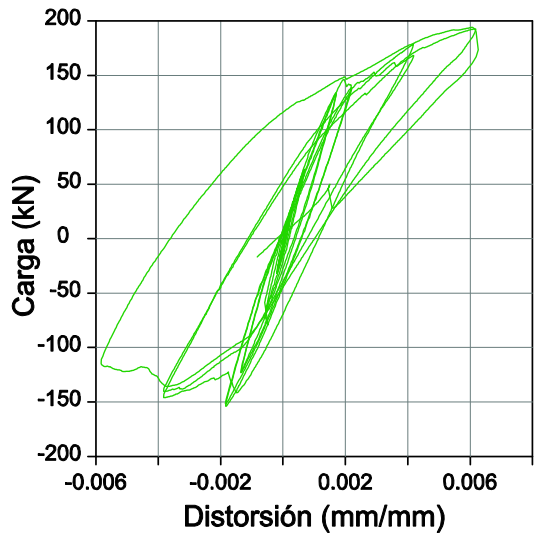

Muro M1

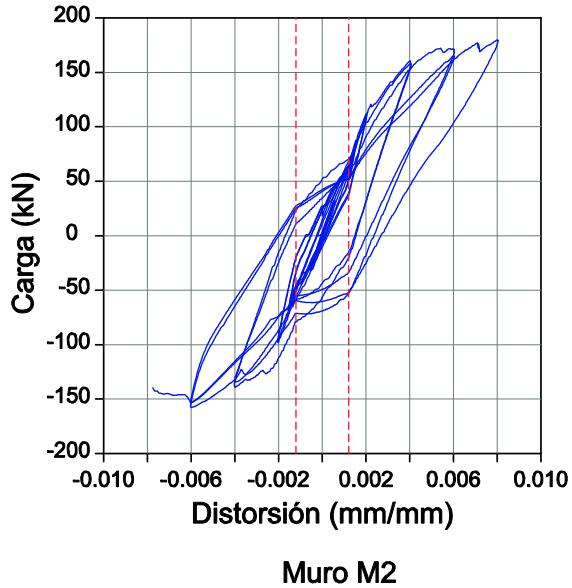

Muro M2

Figura 12. Curvas de histéresis de los muros 
Los ciclos del muro M2 presentan marcados cambios de pendiente, más evidentes en la descarga que durante la carga y que coinciden con la distorsión \pm 0.0012 ( $\pm 3 \mathrm{~mm}$ de desplazamiento). Este comportamiento se debe a la secuencia de carga; recuérdese que el momento se aplica en forma creciente hasta llegar a $\mathrm{M}_{\mathrm{a}}$ cuando se alcanza una distorsión de 0.0012 y en la descarga a partir de este valor distorsión se empieza a reducir el momento aplicado.

\section{Envolventes}

La rigidez "aparente” de la curva envolvente del muro M2 es claramente menor que la rigidez del muro M1 (fig. 13), se emplea el término "aparente” ya que los desplazamientos laterales en el muro M2 son causados tanto por cortante como por momento. Se ha marcado con línea punteada la distorsión a la que termina de aplicarse el momento $M_{a}$ y se observa que se cumplió la expectativa de que el agrietamiento se presentara después de este punto. En el tramo de cero a una distorsión de 0.0012 la curva de M2 tiene una pendiente ligeramente menor a la pendiente del tramo de 0.0012 al agrietamiento, la pendiente de este segundo tramo es parecida a la del muro M1, ya que este segundo tramo fue generado solo por fuerza cortante. Este efecto es mucho más evidente durante la descarga en las curvas de histéresis. Como se había predicho el cortante de agrietamiento fue menor en el muro M2. El cortante máximo también se redujo en una proporción similar, sin que exista la posibilidad de generar una predicción al respecto con el modelo matemático desarrollado ya que está fuera del rango lineal.

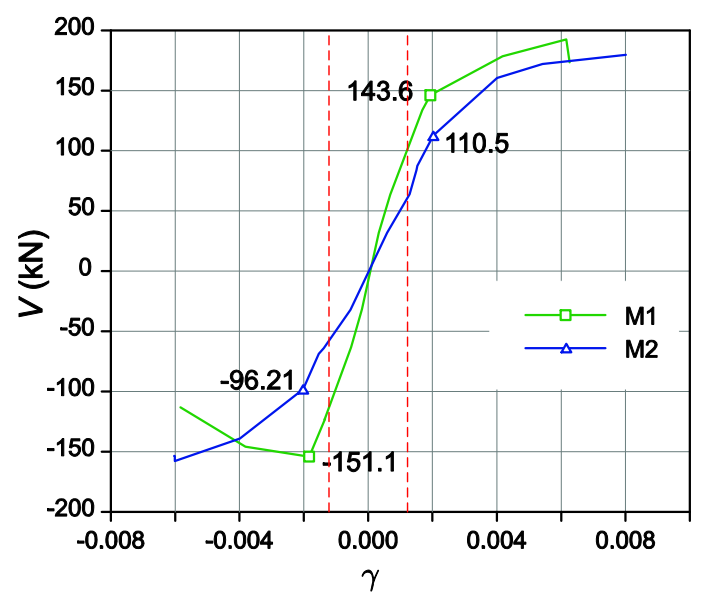

Figura 13. Comparativa de envolventes

En la curva del muro M2, antes del agrietamiento se aprecian pequeños quiebres que corresponden a agrietamientos menores que no tuvieron un efecto importante en el valor del cortante. En cuanto a la hipótesis de que el agrietamiento con y sin momento se presentará al mismo nivel de deformación, se observó, que en la rama positiva, el agrietamiento en el muro con momento se presentó a una distorsión $4.8 \%$ mayor que en el muro sin momento, mientras que en la rama negativa fue $9.9 \%$ mayor. El primer agrietamiento fue, tanto en el muro M1 como en el M2 en la rama positiva, por lo que la desviación mayor en la rama negativa respecto al modelo matemático se puede explicar en parte a que el muro ya tenía un cierto nivel de daño.

\section{Degradación de rigidez}

En esta sección se utiliza la rigidez de ciclo y la deformación promedio de ciclo que se definen como 
$k_{\text {ciclo }}=\frac{V^{+}-V^{-}}{d^{+}-d^{-}} \quad \gamma_{\text {ciclo }}=\frac{d^{+}-d^{-}}{2 H}$

donde $V^{+}$y $V^{-}$son las fuerzas cortantes positiva y negativa máximas del ciclo, $d^{+}$y $d^{-}$los desplazamientos a los que se presentan dichas fuerzas y $H$ la altura del muro.

Las curvas de degradación de rigidez con la deformación de los muros M1 y M2 se presentan en la fig. 14.a. El comportamiento que se observa nos indica que cuando el muro se deforma por efecto de cortante y momento la degradación de la rigidez es significativamente menor. Este efecto es independientemente de que la rigidez "aparente" de los muros que con flexión es menor. Este comportamiento puede atribuirse a que el momento tiende a aumentar los esfuerzos en los extremos del muro normal a las juntas mientras que el cortante produce distorsión de las piezas y por lo mismo es potencialmente más destructivo. Estas curvas son una forma de caracterizar el nivel de daño en el muro.

\section{Energía disipada}

La energía disipada en un ciclo corresponde al área contenida en un ciclo de la curva de histéresis y se calcula como

$E_{d}=\frac{\sum_{i=1}^{n} f_{i}\left(d_{i+1}-d_{i-1}\right)}{2}$

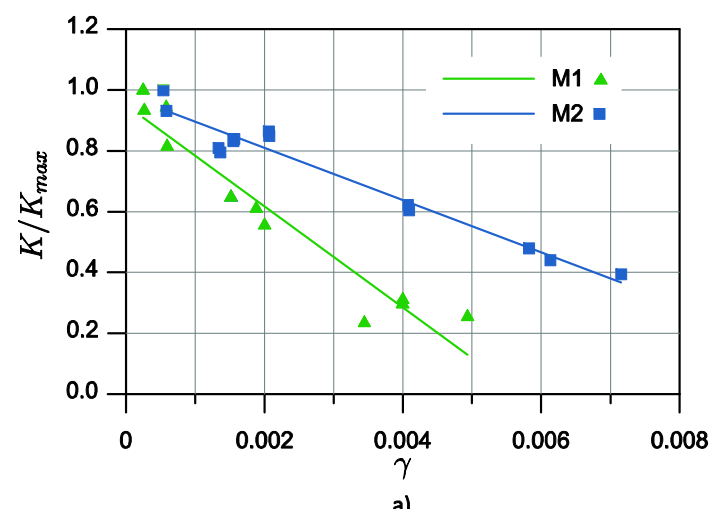

a)

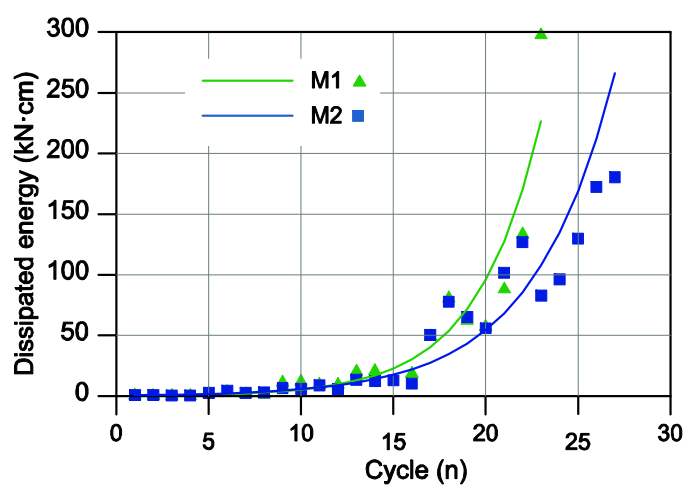

b)

Figura 14. a) Degradación de rigideces y b) energía disipada acumulada

donde $\left(d_{i}, f_{i}\right)$ es el $i$ esimo punto de la curva de histéresis. La disipación de energía acumulada de los muros M1 y M2 se muestra en la fig 14.b. Como en el caso de la degradación de rigidez, estas curvas están correlacionadas con el nivel de daño en el muro. Se puede interpretar que el daño de los muros sin momento flexionante en su extremo superior en el que la distorsión es debida en mayor medida a corte, el daño es mayor para el mismo número de ciclos.

\section{Deformación por cortante y flexión}

La fig. 15 muestra las componentes de deformación del muro, respecto del total. Como era de esperarse las deformaciones por cortante son menores en el caso del muro M2. La relación entre la deformación por corte y momento en el muro sin momento flexionante puede verificarse analíticamente y coincide bien con la medición que se presenta. En ambos casos la proporción de deformación por cortante crece cuando empiezan a agrietarse el muro, después del $4^{\circ}$ ciclo. 

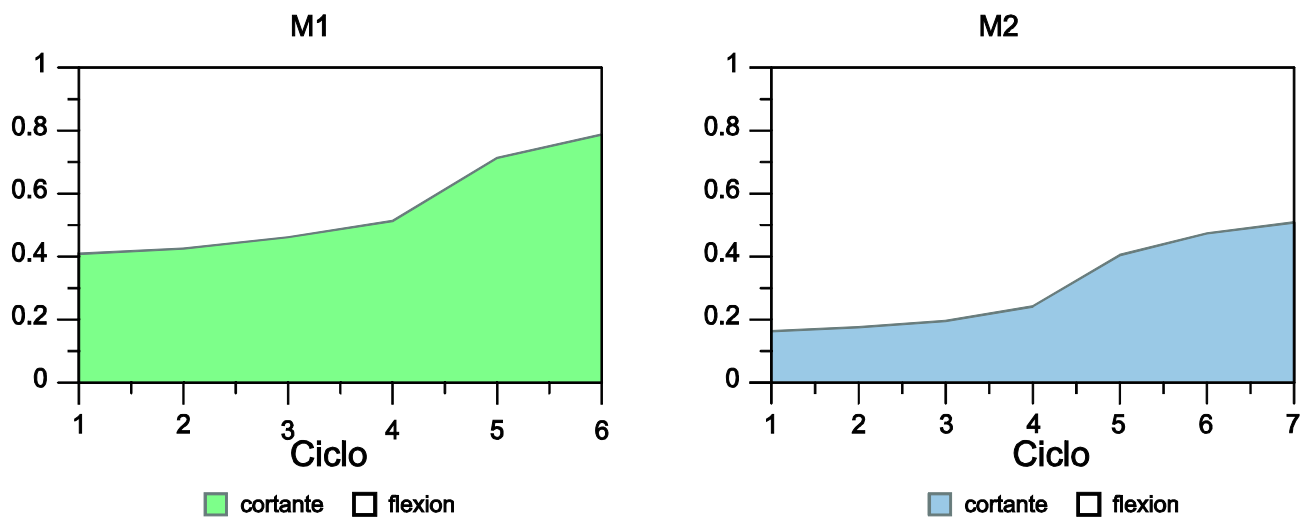

Figura 15. Contribución de la deformación por corte y flexión respecto al total

\section{Interacción cortante momento}

En la evaluación, se tomó como resistencia nominal $V_{n 1}$ el valor de la carga de agrietamiento del muro M1, probado sin momento flexionante y la carga de agrietamiento $V_{n 2}^{\prime}$, al valor registrado de la carga lateral cuando se produjo el agrietamiento del muro M2, probado con momento $M_{a 2}=176.52 \mathrm{kN}$ m. Se calculó el cociente $\alpha=V_{n 2}^{\prime} / V_{n 1}$ y se comparó con los que predice la ecuación (7). Para el cálculo del parámetro $\eta$ se emplearon los valores promedio de $G$ y $E$ de todas las pilas probadas y el parámetro $\beta$ como $\beta=M_{a 2} /\left(V_{n 2}^{\prime} H / 2\right)$.

El cortante al agrietamiento del muro sujeto a momento flexionante de $176.52 \mathrm{kN}-\mathrm{m}$ fue el $77 \%$ del que se obtuvo cuando no tenía momento $\left(V_{n}^{\prime}=0.77 V_{n}\right)$. Con la expresión desarrollada el valor del cortante al agrietamiento se esperaba $V_{n}^{\prime}=0.71 V_{n}$, esto es el error relativo de la predicción considerando al resultado experimental como referencia fue de $-7.8 \%$. $\left(e=\frac{0.71-0.77}{0.77} \times 100=-7.79\right)$. Los resultados se presentan en la tabla 4.

Tabla 4. Cortante nominal y reducido por la interacción cortante momento

\begin{tabular}{lcc}
\hline & M1 & M2 \\
\hline$M_{a}(\mathrm{kN}-\mathrm{m})$ & 0.0 & 176.5 \\
$P(\mathrm{kN})$ & 392.3 & 392.3 \\
$V_{n}(\mathrm{kN})$ & 143.6 & 143.6 \\
$V_{n}^{\prime}$ & & 110.5 \\
$\beta=M_{a} /\left(V_{n}^{\prime} H / 2\right)$ & 0 & 1.3 \\
$\alpha$ (calculado) & 1 & $\mathbf{0 . 7 1}$ \\
\hline$\alpha$ (experimental) & 1 & $\mathbf{0 . 7 7}$ \\
\hline
\end{tabular}

\section{Comparación con el código de Perú}

Para comparar la propuesta que se presenta en esta contribución con la expresión del reglamento peruano, es posible expresar la ec. 2 antes de ser afectada por el factor correctivo $V L / M$ como

$\frac{V_{n}}{A v_{m}}=a+b s$ 
donde $s=P / A v_{m}=\sigma / v_{m}$ y $a, b$ son constantes. Después de afectar por el factor $V L / M$

$\frac{V_{n}^{\prime}}{A v_{m}}=a \cdot \frac{V L}{M}+b s$ obtiene

Si se sustituye $M=M_{a}+V H, M_{a}=\beta V H / 2$ y se toma el cociente de las ecuaciones (16) y (15) se

$\frac{V_{n}^{\prime}}{V_{n}}=\frac{10+3 s w+6 s w}{w(\beta+2)(5+3 s)}$

momento y la relación de aspecto del muro del reglamento peruano, equivalente a la ec. 8 y en términos de los mismos parámetros excepto que la expresión obtenida para el reglamento peruano depende de ' $s$ ' que es el nivel de carga axial normalizado con el esfuerzo cortante resistente de la mampostería. En la fig. 16 se presenta la comparación de las ecuaciones 6 y 7 en función de la cantidad de momento o condición de frontera del extremos superior del muro representada por $\beta$; en a) cuando $w=1$ y dos niveles de carga axial $s=0.5$ y $s=1.0$ y en b) cuando $w=1.5$ para los mismos niveles de carga.

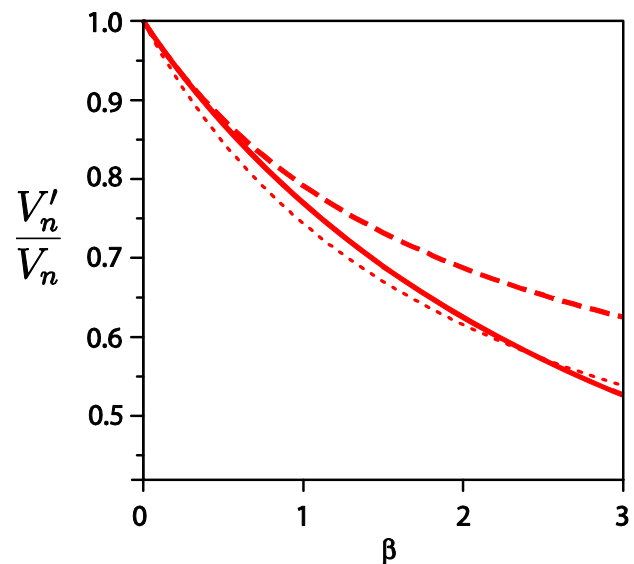

a) --- Perú $\sigma / v^{*}=1.0 \quad w=1$

…. Perú $\sigma / v^{*}=0.5 \quad w=1$

- Propuesta $\eta=0.2 w=1.0$

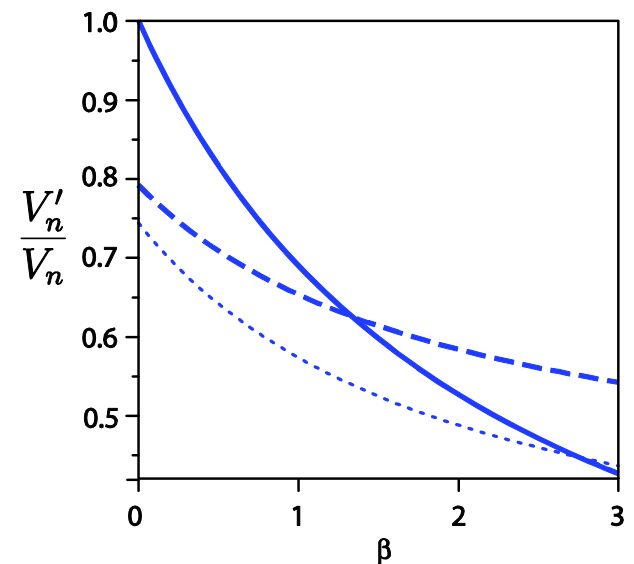

b) - - Perú $\sigma / v^{*}=1.0 \quad w=1.5$

$\cdots$ Perú $\sigma / v^{*}=0.5 \quad w=1.5$

Propuesta $\eta=0.2 w=1.5$

$$
\begin{aligned}
& \text { Propuesta } \\
& \frac{V_{n}^{\prime}}{V_{n}}=\frac{1}{1+\frac{15 \beta w^{2}}{20 \eta w^{2}+6}}
\end{aligned}
$$

Perú

$$
\frac{V_{n}^{\prime}}{V_{n}}=\frac{10+3 s w \beta+6 s w}{w(\beta+2)(5+3 s)} \quad s=\frac{\sigma}{v^{*}}
$$

Figura 16. Comparación de la propuesta en este artículo y el reglamente peruano, para dos niveles de carga axial. a) para $w=1$, b) para $w=1.5$

La ec. 17 es la expresión que predice la reducción del cortante resistente nominal en función del Se observa en a) que la propuesta de esta contribución es parecida a la peruana si el nivel de carga es $s=0.5$ y que el reglamento peruano reduce menos la resistencia a corte si la carga axial es mayor. Esto se debe a que el término de la carga no está afectado por el factor de reducción y a medida que la carga axial es mayor, esta representa una mayor proporción de la resistencia. En b) cuando el muro es más esbelto $(w=1.5)$ el reglamento peruano predice una reducción aun cuando el muro esta en voladizo $(\beta=0)$; dicha predicción contradice lo observado experimentalmente (fig. 1). La predicción que aquí se propone 
no reduce la resistencia cuando no hay momento en el extremo superior del muro, es menos conservadora que el reglamento peruano hasta cierto punto: en muros con elevada carga axial el código peruano reduce menos la resistencia.

\section{CONCLUSIONES}

Se pueden listar las siguientes conclusiones

- En base a las observaciones y mediciones hechas durante los ensayes puede concluirse que la carga de agrietamiento sufre una disminución en presencia del momento flexionante, del orden del 23\%. La razón entre cortantes obtenidos experimentalmente con y sin momento, presenta una variación con la predicción numérica del $8 \%$.

- La hipótesis de igual desplazamiento se cumplió satisfactoriamente ya que el agrietamiento en cada muro ocurrió a niveles prácticamente iguales de distorsión (0.00193 para M1 y 0.00203 para M2), con una variación del 5\%

- La degradación de rigidez y la energía disipada acumulada, muestran que el daño acumulado es más severo cuando las distorsiones son debidas, principalmente a fuerza lateral, en comparación con las producidas por flexión y cortante.

- De la fig. 4.a se concluye que el cortante resistente de muros esbeltos puede reducirse hasta en un $55 \%$ para niveles elevados de momento $(\beta=2)$

- La expresión propuesta para predecir la resistencia cortante por tensión diagonal ec. 7, permite identificar el efecto de la relación de aspecto y el momento flexionante en el extremo superior de muro, en forma independiente.

- La expresión ec. 7 que se propone no reduce la resistencia con la relación de aspecto de muros que no tienen un momento flexionante en su extremo superior, consistentemente con las observaciones experimentales presentadas en otros estudios.

- La expresión de las NTCM podría actualizarse para incluir el efecto de la flexión en la resistencia al agrietamiento, adoptando la forma siguiente:

$$
V_{m R}=F_{R}\left(0.5 v^{*} A_{T}+0.3 P-\frac{M_{a}}{H_{k}}\right)
$$

Aunque los resultados son satisfactorios deben verificarse, con un programa experimental, el efecto de los parámetros, $\eta=G / E, w=H / L$ y $\beta=V_{n}^{\prime} H / 2$, haciendo énfasis en los dos últimos. Debe además de seleccionarse un diseño de los muros que permita un mayor contraste entre los valores de cortante al agrietamiento en presencia y ausencia del momento flexionante.

\section{SÍMBOLOS UTILIZADOS}

A Área transversal del muro incluyendo los castillos $A=t L$

E Módulo de elasticidad

$E_{d} \quad$ Energía disipada hasta el agrietamiento

G Módulo de cortante de la mampostería

$H \quad$ Altura del muro (altura de entrepiso)

$H_{e} \quad$ Altura efectiva $H_{e}=M / V$

$H_{K} \quad$ Propiedad que depende de la geometría y las propiedades de los materiales del muro que tiene unidades de longitud. Puede interpretarse como una altura característica

I Momento de inercia de la sección transversal del muro respecto a un eje centroidal 
paralelo a la menor dimensión del muro.

$k_{f} \quad$ Rigidez a flexión del muro en voladizo

$k_{v} \quad$ Rigidez a cortante de un muro en voladizo

$K_{0} \quad$ Rigidez lateral teórica del muro

$L \quad$ Longitud total del muro

$M, M_{b} \quad$ Momento en la base del muro

$M_{a} \quad$ Momento en el extremos superior del muro

$M_{e} \quad$ Momento que forma parte de un unto del diagrama de interacción

$M_{v} \quad$ Momento que forma parte de un punto del diagrama de interacción modificado, $M_{v} \leq M_{e}$

$P \quad$ Carga axial de compresión en el muro

$P_{e} \quad$ Carga axial que forma parte de un punto del diagrama de interacción

$P_{m} \quad$ Carga axial máxima que produce un aumento en la resistencia nominal a corte.

$P_{\max } \quad$ Carga máxima nominal según las NTCM

$t \quad$ Espesor del muro

$v_{m} \quad$ Esfuerzo cortante resistente nominal de la mampostería obtenida de pruebas de

compresión diagonal

$v^{*} \quad$ Esfuerzo cortante resistente de diseño de la mampostería

$V_{n} \quad \begin{aligned} & \text { Cortante nominal para el que } \\ & \text { el efecto del momento } M_{a}\end{aligned}$

$V_{n}^{\prime} \quad$ Cortante nominal que produce el agrietamiento cuando el muro está sometido a un

momento $M_{a}$ en el extremos superior del muro.

$w \quad$ Relación de aspecto $w=H / L$

(c) ${ }_{r}^{S} \quad$ Cortante o desplazamiento $c=\{V \mid d\}$ al agrietamiento, máximo o último $r=\{c|\max | u\}$

de la rama positiva o negativa de la envolvente $s=\{+\mid-\}$

$\alpha \quad$ Parámetro en la expresión del código peruano $\alpha=V L / M$

$\alpha \quad$ Relación de cortantes nominales resistentes con $\mathrm{y}$ sin considerar el momento $M_{a}$,

$\alpha=V_{n}^{\prime} / V_{n}$

$\delta_{M} \quad$ Desplazamiento lateral debido al momento $M_{a}$

$\delta_{V} \quad$ Desplazamiento debido a una carga lateral $V_{n}^{\prime}$

$\delta_{0} \quad$ Desplazamiento teórica para el cual se presenta el agrietamiento por tensión diagonal y que corresponde al producido por una fuerza lateral $V_{n}$

Parámetro que caracteriza la condición de frontera y cantidad de momento en el extremo

$\beta \quad$ superior del muro.

$\eta \quad$ Relación entre el módulo de cortante y de elasticidad $\eta=G / E$

$\kappa \quad$ Factor de forma de cortante

$(\gamma)_{r}^{S} \quad$ Distorsión al agrietamiento, cuando se presenta el cortante máximo y a la falla o último $r=\{c|\max | u\}$ de la rama positiva o negativa $s=\{+\mid-\}$

\section{AGRADECIMIENTOS}

El presente estudio fue posible gracias al financiamiento del Gobierno del Distrito Federal convenio No. CT/04/10 y parcialmente a los recursos de CONACYT No. de Convenio 133225. Las pruebas de desarrollaron en el laboratorio de estructuras del Instituto de Ingeniería de la UNAM en CU con la Ayuda del Ingeniero Grandry $\dagger$ a cargo del equipo de control, de Raymundo Mondragón en la Calibración e Instalación del equipo de medición y del personal de soporte del Laboratorio. Se agradece el financiamiento y el apoyo brindados. Se agradecen las observaciones de los dos revisores anónimos que permitieron mejorar considerablemente el manuscrito. 


\section{REFERENCIAS}

Alcocer, S. y R. Meli. (1995) "Test program on the seismic behaviour of confined masonry walls." The Masonry Soc. J. (Boulder) 12, No. 2, 68-76.

Alvarez, J. J. (1996) "Some Topics of the seismic behaviour of confined masonry structures." Eleventh World Conference on Eartquake Engineering. Elsevier Science Ltd.

Bazan, E. (1980) "Muros de Mampostería Ante Cargas Laterales”. Tesis Doctoral, México D. F.: Posgrado de Ingeniería, UNAM

Cervantes, Ruiz, J. y R. Jean Perilliat. (2009) “Diseño de Edificios de Mampostería Altos en Zonas Sísmicas” XVII Congrso Nacional de Ingeniería Sísmica.

Davis, C.L., (2008) "Evaluation of design provisions for in-plane shear in masonry walls", Master of Science Thesis, Civil Engineering, Washington State University.

EC6. Eurocode 6: Design of Masonry Structures --Part 1-1: Common rules for reinforced and unreinforced masonry Structures. European Standards, 2002.

López O., Alfredo. (2011) "Implicaciones estructurales de la construcción de edificios altos de mampostería para vivienda e la Ciudad de México”. Tesis de maestría, México, D.F.: Posgrado de Ingeniería de la UNAM.

Matsumura, Akira. (1988) "Shear Strength of Rainforced Masonry Walls." Proceedings of Ninth World Conference on Earthquake Engineering. Tokyo-Kyoto, Japan, pg. 121 - 126.

Meli, R. (1973) "Behaviour of Masonry Walls Under Lateral Loads." Fifth World Conference on Earthquake Engineering. Rome.

Meli, Roberto. (1975) Comportamiento Sísimico de Muros de Mampostería, 2da Ed. Corregida y aumentada. Mexico D. F.: Instituto de Ingeniería de la UNAM.

NTCM. Normas Técnicas Complementarias para Diseño y Construción de Estructuras de Mampotsería. Mexico: Gobierno del Distrito Federal, 2004.

Pérez Gavilán, J. J. (2004) Alinreg, un programa para la alineación, filtado y decimado de registros, (Visual $C++$ con MFC). Instituto de Ingeniería, CU.

Perez Gavilan, J. J., L. E. Flores, y S. M. Alcocer. (2012) “An experimental study of confined masonry walls with varing aspect ratio.” Earthquake Spectra, 2012: (enviado).

Pérez-Gávilan, J. J., L Flores, O Cruz, y P Olalde. (2010) Ensayes de muros de mampostería confinada de piezas de barro extruido multiperforadas: siete muros con distinta relación de aspecto, un muro en ' $T$ ' y dos muros con aberturas. Mexico D. F.: Sociedad Mexicana de Ingeniería Estructural (SMIE).

Pérez-Gavilán, J. J., y A. Manzano. (2012) Interacción Momento Cortante en Muros de mampostería confinada: Un estudio piloto. Series de Investigación y Desarrollo (en revisión), Instituto de Ingeniería.

SEDESOL. Guía para la Redensificación Habitacional de la Ciudad Interior. México D. F.: CONAVI, 2010.

Voon, K. C. y J. M. Ingham. (2006) "Experimental In-Plane Shear Strength Investigation of Reinforced Concrete Masonry Walls." Journal of Structural Engineering, pp. 400-4008.

Zeballos, A., A. San Bartolomé, y A. Muñoz. (1992) "Efectos de la esbeltez sobre la resistencia a fuerza cortante de los muros de albañilería confinada. Analisis por elementos finitos.” Blog de Angel San 
Bartolomé, Pontificia Universidad Católica de Perú, http://blog.pucp.edu.pe/media/688/20070504Esbeltez\%20-\%20Elementos\%20finitos.pdf.. 